\title{
'I couldn't even talk to the patient': Barriers to communicating with cancer patients as perceived by nursing students
}

\begin{abstract}
Communication is closely related to safe practice and patient outcomes. Given that most clinicians fall into routines when communicating with patients, it is important to address communication issues early. This study explores Taiwanese nursing students' experiences of communication with patients with cancer and their families. Senior nursing students who had cared for cancer patients were recruited to participate in focus group interviews. These semi-structured interviews were recorded and transcribed for content analysis. Among the 45 participants, about $36 \%$ of them never received any communication training. Up to $76 \%$ of the participants stated that their communication with cancer patients was difficult and caused them emotional stress. Subsequent data analysis revealed four themes: dis-engagement, reluctance, regression, and transition. Students' negative communication experiences were related to the patients' terminally ill situation; the students' lack of training, low self-efficacy and power status, poor emotional regulation, and cultural considerations. The findings of this study provide a deeper understanding of nursing students' communication experiences in oncology settings within the cultural context. Early and appropriate communication training are necessary to help students regulate their emotions and establish effective communication skills. Further studies are needed to examine the relationship among students' emotional labor, communication skills and outcomes.
\end{abstract}




\section{Introduction}

Communication plays a significant role in health care and is important to health education. Although evidence has shown a strong relation between good communication and improved patient outcomes, including emotional health, symptom resolution, function, physiologic measures, pain control and adherence, miscommunication can result in medical errors, extra financial costs and negative attitudes towards health care providers (Fallowfield and Jenkins, 1999; Frydenberg and Brekke, 2012; Griffey and Bohan, 2006; Stewart, 1995; Thorne et al., 2005). Health communication can be particularly challenging in cancer care because cancer is closely linked to a poor prognosis, limited curative choices and increased emotions (Kruijver et al., 2000; Lichtman

et al., 1988; Thorne et al., 2005; Thorne et al., 2004). Furthermore, studies have noted that a considerable number of cancer patients are not satisfied with the overall communication process with nurses (Suominen et al., 1995; Nayak et al., 2005). Nursing experts have attempted to address these communication issues by designing and delivering communication training for nurses and nursing students; however, there is no consensus on essential content and training outcomes. Scant research has explored nursing students' communication experiences with cancer patients, particularly nurses' own emotional reactions.

In addition to the aforementioned communication challenges, nursing students encounter other unique tasks when communicating with cancer patients and their families. Although there is limited evidence, researchers have indicated that these tasks may be related to status, power, knowledge, skills and culture. First, nursing students have lower statuses and less power in the medical environment. Consequently, nursing students often feel oppressed, lacking courage and power in clinical situations (Kav et al., 2013; Yeh et al., 2010). Second, students are overwhelmed by their clinical responsibility to utilize therapeutic communication skills (Thomas and Burk, 2009; 
Sheu and Mu, 2009; Sheu et al., 2002). Third, as culture and health cannot be separated (Helman, 2007), nursing students in Eastern cultures experience special cultural concerns when communicating with cancer patients and their families. These culture-related issues include family-centred care patterns, attitudes towards truth-telling, decision-making and end-of-life care preferences (Kagawa-Singer and Blackhall, 2001; Sung, 1991) can be very different than what nursing students learned from text book written based on Western culture. Another important concept regarding interactions with cancer patients is emotional labour, which was first defined in sociology by Hochschild (1983) as "the management of feeling to create a publicly observable facial and bodily display" (Hochschild, 2012). The regulation of emotion requires effort and training and is considered a professional necessity in nursing. Emotional labour is linked to the quality of communication, interpersonal relationships and nursing care (Mark and Mann, 2005; Gray, 2009). Nevertheless, emotional labour has received insufficient attention in studies and education addressing health communication.

Understanding nursing students' perceptions and experience is necessary for providing appropriate communication training and oncology education (Chant et al., 2002). This study seeks to explore communication barriers encountered by Taiwanese senior nursing students when caring for cancer patients.

\section{Methods}

This is a qualitative descriptive study using focus group interview and content analysis to collect and analyse data. Qualitative descriptive design was selected to accurately describe the phenomenon and represent the meanings of the data (Sandelowski, 2000). Focus group interview was used to explore both individual and shared experiences through interaction and debates (Acocella, 2012). It is an ideal tool to explore underexplored topics and participants (Liamputtong, 
2011).

\section{Participants}

Junior and senior students were recruited from three 4-year baccalaureate nursing programmes in Taiwan. All programmes use a unified curriculum which contains 6-hours in-class communication training and 540-hours clinical practice requirement for fundamental nursing, maternal-child nursing, and adult nursing. The inclusion criteria were that participants must completed 6-hours in-class communication training, have participated in clinical practice, and cared for cancer patients for at least 48 hours prior to participation.

\section{Data collection}

After obtaining IRB approval, participants were recruited for a focus group facilitated by a PHD prepared nurse with expertise in group dynamics and counselling. Eight focus groups, each with 4 to 7 students, were held. Before each group, a demographic questionnaire related to participants' learning experiences of communication skills was completed. Four open-ended questions were used to stimulate the group discussion (Table 1). Each session was videotaped and lasted 60 to 100 minutes. The facilitator encouraged the participants to express, share and discuss their communication experiences with cancer patients.

\section{Data analysis}

There are four steps of content analysis: (1) content selection, (2) selection of the unit of analysis, (3) condensation, and (4) abstraction (Baxter, 1991; Elo and Kyngäs, 2008; Graneheim and Lundman, 2004). By following these four steps, three researchers independently analysed the eight transcribed focus-groups using Nvivo software (QSR International Pty Ltd. Version 8, 2008). The process of condensation yield 67 codes which were than grouped into 33 categories. Finally, four themes and 10 subthemes emerged. Table two presents the example themes, sub-themes, tree 
codes, nodes and narrative. The consistency among three coders was $88 \%$ and assured by subjecting the data to peer debriefing and confirmation by conducting member checks with 5 participants.

\section{Results}

Forty-five students consented to participate in the focus groups. To maintain respondent confidentiality, the actual names of schools and students are not included in this study. Thirty-eight participants were female, and their ages ranged from 20 to 22 years old (Table 3). More than half of the students had read the related communication articles (51\%) and had participated in communication courses (64\%) before being recruited to this study. Students had experience in clinical practice with patients suffering from gastric cancer, bladder cancer, colon cancer, oesophageal cancer, and breast cancer. Seventy-six percent of the students reported that their communication experiences with cancer patients were difficult and caused emotional distress.

Although participants described both negative and positive experiences, they focused more on negative experiences, including failing to initiate a conversation, being unable to maintain a conversation with patient, and being incapable of dealing with difficult conversations. Overall, the four themes that revealed experiences with communication were disengagement, reluctance, regression and transition. Detailed information and examples of each theme and their corresponding subthemes are discussed in the following paragraphs and table 4.

\section{Disengagement}

Disengagement occurred when nursing students felt blocked and unable to approach cancer patients. Students could not initiate communication, a behaviour which prevented the chance to build a relationship. The theme was generated from two subthemes: escaping and boundary keeping. 
Escaping. Rather than trying to break the communication block, students run away from the conversation topics and avoided approaching patients again.

'He (patient) answered simply and briefly; eyes closed, and waved his hands asking me to leave... I felt embarrassed... After that, I was afraid to approach him...afraid of being rejected again.' (A012)

'I didn't feel comfortable entering oncology wards... the depressing atmosphere... and the sight of my hepatica cancer patient, with his mouth half open and being so fragile... I just wanted to run away.' (C008)

'I felt uncomfortable opening a discussion of sexual issues, particularly because the patient was such an elderly person that I did not know how to start.' (B014)

The terminally ill atmosphere or the interpersonal tension generated by patients' verbal and nonverbal cues hindered students' attempts to approach cancer patients. These factors influenced their confidence and caused hesitation in further communicating with the patient. The tension resulted in a desire to escape from the communication by avoiding the patient. Maintaining boundaries. Some of the communication block were caused by caregivers' protection behaviours. These protection behaviours made approaching patients extremely difficult for students.

'The nurse's aide (who is hired by the family) answered the question I asked the patient as if she were the representative. Then she changed the subject without noticing the patient was trying to say something slowly. I couldn't even talk to the patient.' (A005) 
'Because of frequent vomiting, my patient wouldn't take the medicine. She only waved her hands to express her refusal... However, her family strongly insisted that she take the medicine... I disagreed with the family since it was against the patient's will, but I didn't know how to explain it and persuade the family not to force her again.' (B013)

Family plays an important and unique role in caring for patients in Asian culture. The social norms regarding the power and obligations of taking care of illness in the family greatly affect family's attitude toward care and communication and health care provider's practice. Students must cope with negative displays of this kind of power or authority. In these circumstances, students' first attempts at communication were marginalized by these boundaries.

\section{Reluctance}

As nursing students endeavoured to initiate communication to build relationships with cancer patients, they were rejected. Patients and families thought students were too young to understand their situation or withdrew despite student's behaviours. Students were barred in achieving a therapeutic relationship. Students adopted a passive mode, awaited acceptance and were reluctant to communicate. Two subthemes were identified: devaluation and rejection.

Devaluation. Students failed to continue communication as patients or families did not trust or view them as health care professions. Students sensed patients and families' distrust from their nonverbal behaviours.

'He shrugged my hands off with an unfriendly attitude as though I were not qualified to take care of him.' (A001)

Ifelt so scared of the family; she always kept watching me while I conducted every step of care for the patient.' (C002). 
'The forty-something male patient was positive but had a fear of physical pain. He would not let me change the wound dressing because of my gender and age. And I did not have much communication with him after that.' (B003)

Students experienced being treated as novices by patients or families who prevented student involvement in nursing interventions. The students expressed feelings of being devalued as unskilled or untrustworthy.

Rejection. Nursing students faced patients' or families' explicit verbal or non-verbal rejection. The rejection resulted in discouraging further communication.

'He lay on the bed and seemed so moody, and he put his arms on his chest with his eyes closed. He did not want to talk at all and I stood beside his bed for a while. Then, the person with him said, you just go out and do not talk to him today.' (B014)

'The cancer patient was receiving the $3 r d$ or 4th course of chemotherapy; he seemed to know more about the treatment than I did. He refused my hands on him with a bad attitude. In his opinion, my care for him was unnecessary.'(C014)

The patient-student relationship was undermined with some families claiming dissatisfaction with the interaction.

\section{Regression}

Although students had established superficial relationships using basic communication skills, the students encountered other barriers to communication. Difficulties in applying advanced skills or patients' negative perceptions hindered students from further understanding patients' concerns or providing emotional support. Three subthemes were identified: disconnectedness, fear of losing 
control, and avoiding a taboo.

Disconnectedness. Students failed to explore patients' inner perception or respond to patients and caregivers' needs.

'When families asked me, 'This disease is serious, right?' I didn't know how to answer, especially in front of the patient...so I said something impractical, trying to give them some hope.'(B012)

'I couldn't guide the patient to disclose her inner feelings to me. Sometimes she answered my questions briefly and sometimes she just changed the subject. When I tried to discuss the issue of intimacy and sexual relationships after a mastectomy, she ignored me.'(A008)

The nursing students were equipped with basic communication skills; however, in the clinical environment, they struggled to perform and failed to achieve an understanding with patients. The student-patient relationship fluctuated because the students were unsuccessful in communicating despite their self-expectations of success. Although the students had learned that phrases such as "cheer up" would not be helpful in comforting patients, they could not help but use such statements to avoid embarrassment. Such inappropriate communication resulted in an apparent disconnect between students and patients. The students were incapable of probing or directing focused communication when patients tried to change topics.

Fear of losing control. When students tried to manage the communication as a profession, they also needed to deal with their own emotions which affected their communication ability.

'I'm afraid of losing control of the situation if I do something. While receiving the 
diagnosis result, the patient's wife cried... They seemed to lose hope in life. I didn't know how to comfort them, couldn't find the words to say... I felt sad.' (B006)

'It was so tough at the time when the patient was not informed of his diagnosis. He cried out after hearing the bad news, and I also cried for not knowing how to soothe him.' (A010)

'Only if I insisted, then she replied simply and then was silent. I took care of her for 3 weeks, but I do not think that we had a therapeutic relationship. I had no idea how to improve this; I felt that I might have needed to give up hope.' (C013)

When encountering the emotion-laden responses of patients or family members, the students were frightened and worried about losing control of such situations. The students tended to view a patient's crying as a negative behaviour that triggered the students' uneasiness and self-blame. The students reflected that they had failed to perceive patients' psychological cues and need for comfort and reassurance. Consequently, the students struggled to provide care and responded passively.

Avoiding a taboo. Students identified communication topics, such as death, need to be handled with extra cautious.

'It was difficult to approach his inner world. He was in the terminal cancer stage, and no one was with him. It was challenging for me to connect with him.'(C009)

'The patient didn't talk much under the oppressive atmosphere in the room. It forced us to leave immediately after finishing the routine work since we couldn't start a conversation about death.'(C004) 
'A cancer patient said, "I am not going to die... You all want to watch me die!" When he mentioned the word "die", I just did not know how to deal with it.' (A011)

Students recognized that speaking about death in Taiwan is a controversial taboo that renders communication regarding terminal illness difficult. Nursing students with limited end-of-life care experiences encountered complex barriers surrounding denial or unpreparedness regarding terminal diagnoses. Thus, death became a hidden agenda between patients and students.

\section{Transition}

Students improved their communication skills by identifying previous communication barriers and failures. The students were able to conduct in-depth conversations with patients in challenging situations; however, these nurses remained overly sensitive to patients' perceptions of them in the aftermath of previous unsatisfactory interactions. Three subthemes were extracted: dilemma, incapability, and asking for the best.

Dilemma. When communicated with patients and families, students struggled to deliver thoughtful messages to address both patients' and families' needs which sometimes conflict with each other.

'A woman with breast cancer had not disclosed her disease to her children, which made me worry about the possible negative effects on the kids afterwards. However, she insisted in not telling them... I didn't know what I should do.'(C005)

'Some families do not want to let the patients know their condition. I felt helpless due to my limited caring experience... I had no idea what I should or shouldn't have said. My mentor even expected me to figure out how much the patient knew, which scared me. I 
was afraid to spill out the truth to the patient.' (A013)

Students were hesitant to address ambivalent situations, including breaking bad news or telling the truth. In the Taiwanese culture, some families insist that patients are too vulnerable to receive bad news and choose to hide the prognosis from patients. Wishing to respect families' decisions conflicted with notions of patient-centred care and created a dilemma in nursing students. One student reported that when families requested that she conceal the diagnosis, she became anxious regarding the assigned task and cautious regarding her conversations with the patient.

Incapability. Students reflected on their incompetence in regard to communicating with cancer patients and their families.

'After talking to them, I found that they had lots of problems that needed to be solved. My inability to help them made me feel depressed.'(C014)

'I wanted to help but was afraid to hurt the patients. I intended to ask my former patient the differences between before and after surgery or about his emotions. But I held back. I felt restricted and was concerned about my expression and questions.' (B008)

Students considered the possible negative effects on patients during direct communication regarding patients' diagnosis and problems. Their self-reflection barriers and caution impeded continuous communication and care. Students expressed frustration regarding their inability to help patients solve problems.

Asking for the best. The nursing students cautiously modified their communication styles with cancer patients following previous ineffective interactions. 
'The first time I changed her dressing comparatively slower than other times, she was unhappy due to the pain of the wound. I told her that I'd do it faster in order to avoid hurting her tomorrow. I kept my promise, and she returned her gratitude cheerfully with a thumbs-up.'(C012)

I discovered lots of problems with communication. I would feel quite powerless when I could not solve those problems in this week of her life countdown.' (A009)

In response to frustrating experiences, the students attempted to solve problems and determine how best to proceed. For example, when the students observed that their communication resulted in patient displeasure, these nurses tried to remedy the situation by fulfilling the patients' needs and soliciting a more favourable impression.

\section{Discussion}

To our knowledge, this is the first study exploring communication experiences with cancer patients from the perspectives of nursing students in Taiwan. By identifying the four themes of disengagement, reluctance, regression and transition, this study delineated a range of experiences regarding nursing students' interactions with hospitalized cancer patients and their families. It is noteworthy that only the theme of transition is related to positive experience. Obviously, students talked less about their positive communication experiences including successful examples from healthcare providers. Two reasons may contribute to this phenomenon. First, communication is not a learning focus of clinical practice. Moreover, nursing preceptors may not know how to facilitate communication or demonstrate communication skills. Second, communication barriers stimulated more productive discussions and interactions in the focus groups because it is a shared experience. These barriers reduced the nursing students' confidence to initiate and maintain a 
therapeutic relationship with cancer patients. Consequently, the nursing students were passive, afraid and oversensitive during communication. They became hesitant and self-conscious while simultaneously struggling to overcome barriers and develop strategies to enhance communication. The students' communication barriers may be associated with multiple interrelated factors, such as insufficient training, low self-efficacy, poor emotional regulation, student identity, and the special cultural care model.

\section{Insufficient Trainings in Communication Skills}

Although approximately half of the participating students had some form of communication training, the majority of the students mentioned the gap between knowledge and practice as described in the theme of transition. Insufficient training in communication and the knowledge gap are consistent with findings from a literature review (Chant et al., 2002). Furthermore, although half of the students did not receive any formal cancer communication training, their clinical placement forced them to interact with vulnerable cancer patients and families with special communication considerations and needs. Disengagement was the initial obstacle when students tried to maintain clear boundaries between themselves and cancer patients. Their initial communication attempts ended in interpersonal stagnation, which is consistent with a prior study of medical students. Lin and colleagues (2001) observed that skills in approaching patients and initiating conversations were the primary difficulties for medical students (Lin et al., 2001). After an unsuccessful opening, some students fled from the conversation, which is consistent with the findings of Hjörleifsdóttir and Carter (2000). Like registered nurses and medical students, nursing students also experienced communication difficulties regarding special conditions, such as delivering bad news (Supiot and Bonnaud-Antignac, 2008, Malloy et al., 2010). Although it is not students' or nurses' responsibility to deliver bad news, it does not permit them to avoid the social 
pressure of this kind of difficult communication. Nursing students felt trapped between patient autonomy, medical paternalism and family protectionism (Sheu et al., 2006). Our findings suggest that nurses sensed and experienced these communication difficulties early in their careers. Addressing these communication issues early in the student stage, when the nursing students are forming their professional identities and establishing a communication style, may be more appropriate.

Insufficient training in communication skills may be the most obvious and universal problem; however, the lack of training is only one cause of ineffective communication. Our findings revealed a wide variation of nursing students' communication experiences, from completely blocked (disengagement) to conducting more effective communication (transition). We concluded that the blocked status (e.g., disengagement and reluctance) may be more relevant to students' emotional regulation, power status and cultural considerations than communication knowledge and skills. In other words, students must effectively regulate their own emotions before applying their cancer communication knowledge and skills. However, emotional regulation appears to have received less attention in studies examining health communication.

\section{Poor Emotional Regulation}

Our findings showed that nursing students had difficulties in regulating their own and patients' emotions. As described in the themes of disengagement, reluctance and regression, the students tended to escape and avoid emotional situations. They felt significant emotional fluctuation and feared losing control when being devalued or rejected by patients, nursing aides or families. These feelings, in addition to the perceived severity of the topic (e.g., death as a taboo issue) and low feelings of efficacy, impeded students from probing and achieving a deeper mutual understanding with their patients. This finding supports the results of studies conducted in Turkey 
and the United Kingdom that revealed nursing students' fear of addressing emotion and their use of blocking or distancing strategies (King-Okoye and Arber, 2014, Kav et al., 2013). Moreover, considering the different performance levels of emotional regulation, our findings indicated that the students regulated their emotions on a more surface, response-focused level (e.g., escaping from the emotional situation based on patients or families' negative responses) rather than at a deep, antecedent-focused level (Mark and Mann, 2005).

\section{Culture Considerations}

Compared with most Western cultures, health care and communication in cultures such as those in Japan, China and Mexico are more family-focused (Harris and Long, 1999; Hicks and Lam, 1999; Frank et al., 2002). In these cultures, family caregivers believe that they have the responsibility to protect the patients by filtering information and assisting with making decisions (Frank et al., 2002). Similarly, in Taiwan, family caregivers are allowed and expected to provide bedside care and to participate in communication and decision-making. Some Taiwanese families hire nurses' aides for bedside care (Lin, 2000; Sung et al., 2005); these nurses' aides may have more experience and familiarity with cancer patients than nursing students. Similar to other Taiwanese studies that found that families perceive themselves to be decision-makers and gatekeepers who are ethically responsible for safeguarding their vulnerable members (Sheu et al.,

2006; Lin, 2000; Fan and Li, 2004), our subtheme of maintaining boundary delineated how family caregivers participate in or even dominate communication in order to protect patients. Because family members perceived student nurses to be inexperienced health care providers, the family tended to take the lead in the patient-student conversations. It is important for educators and students to understand the meanings and health beliefs behind family-dominant conversations.

Although the involvement of families in patient care is a particularly important family 
centred care model, understanding and responding to families' needs and emotions are always challenging, particularly for students, when caring for terminally ill patients worldwide (Charalambous and Kaite, 2013; Sanford et al., 2011; Huang et al., 2010). Our findings showed the importance of preparing students to communicate with families and recognize their concerns and needs before assigning nursing students to terminally ill patients. Although the majority of textbooks and caring models were developed and tested in Western cultures that focus on individuality, our findings noted the importance of developing culturally appropriate care and education.

Early and comprehensive communication training is imperative. In the short term, such training can reduce the pressure of working in an oncology setting, which can frustrate students and drain their energy (Basso Musso et al., 2008). Over the long term, training enhances students' positive clinical experience, which is positively related to future job satisfaction (Wu and Norman, 2006). Adequate communication training for nursing students thus has the potential to promote positive nurse-patient relationships and enhance nurse retention.

\section{Conclusion}

The findings of the current study provide a deeper understanding of nursing students' communication experiences in Taiwanese oncology settings, further advancing insights into a student's progress from novice to qualified nurse. Unfortunately, our results did reveal that students

experience traumatic communication experiences that prohibit these nursing students from communicating effectively with cancer patients. The lack of preparation, including communication knowledge, skills and emotional regulation, before clinical placement in cancer settings contributes to nursing students' negative communication experiences. Special communication training programmes targeting patient populations with different needs and considerations may be 
necessary for nursing students or novice nurses who will care for unfamiliar patient populations. Moreover, culture-specific considerations such as families' protective behaviour should be identified and discussed openly. Ideally, educators and clinical preceptors must communicate with and educate families before assigning students to patients. Other strategies such as role-playing, case scenarios or exposure in a less stressful environment are recommended to enhance students' feelings of self-efficacy (Sanford et al., 2011). Examples from the current study (e.g., reluctance or regression scenarios) can be used as cases to raise awareness or facilitate discussion.

Identifying emotional reactions and regulating emotions adequately are important abilities when communicating with vulnerable patients and families. Students can be trained in these skills by being guided to recognize and express their communication experiences and feelings in reflective journals or small group discussions. Because emotional labour attracts relatively less attention in studies addressing communication education, future studies are necessary to examine the relations among health care students' emotional regulation, communication ability and communication outcomes. 


\section{References}

Acocella, I. (2012). The focus groups in social research: advantages and disadvantages. Quality \& Quantity, 46(4), 1125-1136.

Basso Musso, L., Ardiles Vargas, B., Bernal Torres, M., Canovas Del Canto, M. J., González Meléndez, C., Kroff Balloqui, M. F. and Soto Cornejo, A. (2008). Factors derived from the intrahospitable laboratories that cause stress in infirmary students. Revista LatinoAmericana de Enfermagem, 16(5), 805-811.

Baxter, L. A. (1991). Content analysis. In B. Montgomery and S. Duck (Eds.), Studying interpersonal interaction (pp. 239-254). NY: New York: The Guildford Press.

Chant, S., Jenkinson, T., Randle, J. and Russell, G. (2002). Communication skills: some problems in nursing education and practice. Journal of Clinical Nursing, 11(1), 12-21.

Charalambous, A. and Kaite, C. (2013). Undergraduate nursing students caring for cancer patients: hermeneutic phenomenological insights of their experiences. BMC Health Services Research, 13(1), 63.

Elo, S., \& Kyngäs, H. (2008). The qualitative content analysis process. Journal of Advanced Nursing, 62(1), 107-115.

Fallowfield, L. and Jenkins, V. (1999). Effective communication skills are the key to good cancer care. European Journal of Cancer, 35(11), 1592-1597.

Fan, R. and Li, B. (2004). Truth telling in medicine: The Confucian view. The Journal of Medicine and Pilosophy, 29(2), 179-193.

Frank, G., Blackhall, L. J., Murphy, S. T., Michel, V., Azen, S. P., Preloran, H. M. and Browner, C. H. (2002). Ambiguity and hope: disclosure preferences of less acculturated elderly Mexican Americans concerning terminal cancer-a case story. Cambridge Quarterly of 
Healthcare Ethics, 11(02), 117-126.

Frydenberg, K. and Brekke, M. (2012). Poor communication on patients' medication across health care levels leads to potentially harmful medication errors. Scandinavian Journal of Primary Health Care, 30(4), 234-240.

Graneheim, U. H. and Lundman, B. (2004). Qualitative content analysis in nursing research: concepts, procedures and measures to achieve trustworthiness. Nurse Education Today, 24(2), 105-112.

Gray, B. (2009). The emotional labour of nursing-Defining and managing emotions in nursing work. Nurse Education Today, 29(2), 168-175.

Griffey, R. T. and Bohan, J. S. (2006). Healthcare provider complaints to the emergency department: a preliminary report on a new quality improvement instrument. Quality and Safety in Health Care, 15(5), 344-346.

Harris, P. B. and Long, S. O. (1999). Husbands and sons in the United States and Japan: Cultural expectations and caregiving experiences. Journal of Aging Studies, 13(3), 241-267.

Helman, C. G. (2007). Culture, Health and Illness: An Introduction for Health Professionals (2nd ed.). London: CRC Press.

Hicks, M. H. R. and Lam, M. S. C. (1999). Decision-making within the social course of dementia: accounts by Chinese-American caregivers. Culture, Medicine and Psychiatry, 23(4), 415452.

Hjörleifsdóttir, E. and Carter, D. E. (2000). Communicating with terminally ill cancer patients and their families. Nurse Education Today, 20(8), 646-653.

Hochschild, A. R. (2012). The Managed Heart: Commercialization of Human Feeling, University of California Press. 
Huang, X. Y., Chang, J. Y., Sun, F. K. and Ma, W. F. (2010). Nursing students' experiences of their first encounter with death during clinical practice in Taiwan. Journal of Clinical

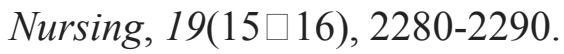

Kagawa-Singer, M. and Blackhall, L. J. (2001). Negotiating cross-cultural issues at the end of life: You got to go where he lives. Jama, 286(23), 2993-3001.

Kav, S., Citak, E. A., Akman, A. and Erdemir, F. (2013). Nursing students' perceptions towards cancer and caring for cancer patients in Turkey. Nurse Education in Practice, 13(1), 4-10.

King $\square$ Okoye, M. and Arber, A. (2014). 'It stays with me’: the experiences of second $\square$ and third $\square$ year student nurses when caring for patients with cancer. European Journal of Cancer Care, 23(4), 441-449.

Kruijver, I. P., Kerkstra, A., Bensing, J. M. and Van De Wiel, H. B. (2000). Nurse-patient communication in cancer care: a review of the literature. Cancer Nursing, 23(1), 20-31.

Liamputtong, P. (2011). Focus group methodology: Principle and practice. Sage Publications.

Lichtman, R. R., Taylor, S. E. and Wood, J. V. (1988). Social support and marital adjustment after breast cancer. Journal of Psychosocial Oncology,5(3), 47-74.

Lin, C. C. (2000). Barriers to the analgesic management of cancer pain: a comparison of attitudes of Taiwanese patients and their family caregivers.Pain, 88(1), 7-14.

Lin, C. T., Barley, G. E. and Cifuentes, M. (2001). Personalized remedial intensive training of one medical student in communication and interview skills. Teaching and Learning in Medicine, 13(4), 232-239.

Malloy, P., Virani, R., Kelly, K. and Munévar, C. (2010). Beyond bad news: Communication skills of nurses in palliative care. Journal of Hospice \& Palliative Nursing, 12(3), 166-174.

Mark, A. and Mann, S. (2005). A health-care model of emotional labour: an evaluation of the 
literature and development of a model. Journal of Health Organization and Management, 19(4/5), 304-317.

Nayak, S., Pradhan, J. P. B., Reddy, S., Palmer, J. L., Zhang, T. and Bruera, E. (2005). Cancer patients' perception of the quality of communication before and after the implementation of a communication strategy in a regional cancer center in India. Journal of Clinical Oncology, 23(21), 4771-4775.

Sandelowski, M. (2000). Focus on research methods-whatever happened to qualitative description? Research in Nursing and Health, 23(4), 334-340.

Sanford, J., Townsend-Rocchiccioli, J., Quiett, K. and Trimm, D. (2011). "I See My Mother's Face": Student nurse experiences caring for cancer patients. European Journal of Oncology Nursing, 15(1), 46-52.

Sheu, S. J. and Mu, P. F. (2009). The nurses' experiences of truth telling to the terminal cancer patients: a systematic review. JBI Database of Systematic Reviews and Implementation Reports, 7(8), 1-8.

Sheu, S., Lin, H. S. and Hwang, S. L. (2002). Perceived stress and physio-psycho-social status of nursing students during their initial period of clinical practice: the effect of coping behaviors. International Journal of Nursing Studies, 39(2), 165-175.

Sheu, S. J., Huang, S. H., Tang, F. I. and Huang, S. L. (2006). Ethical decision making on truth telling in terminal cancer: medical students' choices between patient autonomy and family paternalism. Medical Education, 40(6), 590-598.

Stewart, M. A. (1995). Effective physician-patient communication and health outcomes: a review. CMAJ: Canadian Medical Association Journal, 152(9), 1423.

Sung, H. C., Chang, S. M. and Tsai, C. S. (2005). Working in long $\square$ term care settings for older 
people with dementia: nurses' aides. Journal of Clinical Nursing, 14(5), 587-593.

Sung, K. T. (1991). Family-centered informal support networks of Korean elderly: The resistance of cultural traditions. Journal of Cross-Cultural Gerontology, 6(4), 431-447.

Suominen, T., Leino-Kilpi, H. and Laippala, P. (1995). Who provides support and how? Breast cancer patients and nurses evaluate patient support.Cancer Nursing, 18(4), 278-285.

Supiot, S. and Bonnaud-Antignac, A. (2008). Using simulated interviews to teach junior medical students to disclose the diagnosis of cancer. Journal of Cancer Education, 23(2), 102-107.

Thomas, S. P. and Burk, R. (2009). Junior nursing students' experiences of vertical violence during clinical rotations. Nursing Outlook, 57(4), 226-231.

Thorne, S. E., Bultz, B. D. and Baile, W. F. (2005). Is there a cost to poor communication in cancer care?: a critical review of the literature. Psycho $\square$ oncology, 14(10), 875-884.

Thorne, S. E., Harris, S. R., Mahoney, K., Con, A. and McGuinness, L. (2004). The context of health care communication in chronic illness. Patient education and counseling, 54(3), 299-306.

Wu, L. and Norman, I. J. (2006). An investigation of job satisfaction, organizational commitment and role conflict and ambiguity in a sample of Chinese undergraduate nursing students. Nurse Education Today, 26(4), 304-314.

Yeh, M. Y., Wu, S. M. and Che, H. L. (2010). Cultural and hierarchical influences: ethical issues faced by Taiwanese nursing students. Medical education, 44(5), 475-484. 
Running head: BARRIERS TO COMMUNICATING WITH CANCER PATIENTS

Table 1.

Focus Group Interview Guide

1. Do you think communication is important when caring for cancer patients? Why?

2. Please share positive examples of patient-nurse communications which you have observed or gone through yourself while taking care of cancer patients.

3. Please describe the communication problems that you have encountered in the past. And tell us when, where, and with whom you try to communicate?

4. What were the possible reasons that caused your communication problems? 


\section{BARRIERS TO COMMUNICATING WITH CANCER PATIENTS}

Table 2.

Example Themes, Subthemes, Categories, Codes and Narrative

\begin{tabular}{|l|l|l|l|l|}
\hline Theme & Sub-theme & Category & Code & Narrative \\
\hline Disengagement & $\begin{array}{l}\text { Maintaining } \\
\text { Boundaries } \\
\text { by nurse aid }\end{array}$ & $\begin{array}{l}\text { Being interrupted } \\
\text { ignored patient's } \\
\text { attempt of } \\
\text { communication by } \\
\text { answering questions } \\
\text { for patient }\end{array}$ & $\begin{array}{l}\text { The private nurse aid answered the question I } \\
\text { asked the patient as if she was the } \\
\text { representative. Then she changed the topic } \\
\text { without noticing the patient was trying to say } \\
\text { something slowly. I couldn't even talk to the } \\
\text { patient }\end{array}$ \\
\hline Reluctance & Devaluation & $\begin{array}{l}\text { Fear of being } \\
\text { monitored }\end{array}$ & $\begin{array}{l}\text { Fear of being } \\
\text { monitored by family } \\
\text { members when cared } \\
\text { patient }\end{array}$ & $\begin{array}{l}\text { Ifelt so scared of the family; she just kept } \\
\text { watching me while I did the every step of care } \\
\text { for patient all the time }\end{array}$ \\
\hline Regression & $\begin{array}{l}\text { Fear of Losing } \\
\text { Control }\end{array}$ & $\begin{array}{l}\text { Fear of losing } \\
\text { control due to } \\
\text { patient's and } \\
\text { family's } \\
\text { psychological } \\
\text { reactions }\end{array}$ & $\begin{array}{l}\text { Feel afraid and sad as } \\
\text { being unable to } \\
\text { manage patient's and } \\
\text { family's psychological } \\
\text { reactions }\end{array}$ & $\begin{array}{l}\text { I'm afraid of losing control of the situation if I } \\
\text { do something. While receiving the diagnosis } \\
\text { result, the patient's wife cried... they seemed } \\
\text { to lose hope in life. I didn't know how to } \\
\text { comfort them, couldn't find out the words to } \\
\text { say... I felt sad. }\end{array}$ \\
\hline Transition & $\begin{array}{l}\text { Asking for the } \\
\text { Best }\end{array}$ & $\begin{array}{l}\text { pensitive to } \\
\text { patient's response }\end{array}$ & $\begin{array}{l}\text { Sensed patient's } \\
\text { negative emotion; and } \\
\text { improved patient's } \\
\text { satisfaction by } \\
\text { discussed and } \\
\text { provided solutions to } \\
\text { the problem }\end{array}$ & $\begin{array}{l}\text { The first time I changed her dressing } \\
\text { comparatively slower than others, she was } \\
\text { unhappdue to the pain of the wound. I told } \\
\text { her that I'd do it faster in order to avoid } \\
\text { hurting her tomorrow. I kept my promise, and } \\
\text { she returned her gratitude cheerfully with a } \\
\text { thumb-up. }\end{array}$ \\
\hline
\end{tabular}


BARRIERS TO COMMUNICATING WITH CANCER PATIENTS

Table 3.

Participants' Characteristics $(n=45)$

\begin{tabular}{llc}
\hline Items & & $\mathrm{N}(\%)$ \\
\hline Age range & $20-22$ years & \\
Gender & Male & $7(16 \%)$ \\
& Female & $38(84 \%)$ \\
University & A & $15(33 \%)$ \\
& B & $14(31 \%)$ \\
& C & $16(36 \%)$
\end{tabular}




\section{BARRIERS TO COMMUNICATING WITH CANCER PATIENTS}

Table 4.

Themes and Subthemes Represented the Meaning of Student

Communication Experiences with Cancer Patients

\begin{tabular}{ll}
\hline Themes & Subthemes \\
\hline Disengagement & Escaping \\
& Maintaining boundaries \\
\hline Reluctance & Devaluation \\
& Rejection \\
\hline Regression & Disconnectedness \\
& Fear of losing control \\
& Avoiding a taboo \\
\hline Transition & Dilemma \\
& Incapability \\
& Asking for the best \\
\hline
\end{tabular}

